Comment. Math. Helv. 79 (2004) 758-778

0010-2571/04/040758-21

DOI $10.1007 / \mathrm{s} 00014-004-0810-4$ (c) 2004 Birkhäuser Verlag, Basel

Commentarii Mathematici Helvetici

\title{
Fixity and free group actions on products of spheres
}

\author{
Alejandro Adem*, James F. Davis* and Özgün Ünlü
}

\begin{abstract}
A representation $G \subset U(n)$ of degree $n$ has fixity equal to the smallest integer $f$ such that the induced action of $G$ on $U(n) / U(n-f-1)$ is free. Using bundle theory we show that if $G$ admits a representation of fixity one, then it acts freely and smoothly on $\mathbb{S}^{2 n-1} \times \mathbb{S}^{4 n-5}$. We use this to prove that a finite $p$-group (for $p>3$ ) acts freely and smoothly on a product of two spheres if and only if it does not contain $(\mathbb{Z} / p)^{3}$ as a subgroup.

We use propagation methods from surgery theory to show that a representation of fixity $f<n-1$ gives rise to a free action of $G$ on a product of $f+1$ spheres provided the order of $G$ is relatively prime to $(n-1)$ !. We give an infinite collection of new examples of finite $p$-groups of rank $r$ which act freely on a product of $r$ spheres, hence verifying a strong form of a well-known conjecture for these groups. In addition we show that groups of fixity two act freely on a finite complex with the homotopy type of a product of three spheres. A number of examples are explicitly described.
\end{abstract}

Mathematics Subject Classification (2000). 57S25.

Keywords. Group actions on manifolds.

\section{Introduction}

A well known result in topology is the characterization of those finite groups that can act freely on a sphere, namely groups with either cyclic or generalized quaternion Sylow subgroups and such that every involution is central (see [23]). Free linear actions on spheres are the most basic examples. These can be constructed as follows: a subgroup $G$ of $U(n)$ acts on the homogeneous space $\mathbb{S}^{2 n-1} \cong U(n) / U(n-1)$; if no conjugate of $G$ intersects $U(n-1)$ non-trivially, then this gives rise to a free linear action on the sphere.

In this paper we consider the situation for products of spheres. Here the problem is much more complex, and in particular we still do not have a characterization of those finite groups that can act freely on a product of two spheres. A key new ingredient in our approach is the use of the $G$ actions on the homogeneous spaces $U(n) / U(k)$; our view is that these are also important building blocks for actions

\footnotetext{
${ }^{*}$ The first two authors were partially supported by the NSF.
} 
of groups of larger rank. On the algebraic side, group theorists have studied representations such that the action of $G$ on $U(n) / U(n-f-1)$ is free; the smallest such $f$ is called the fixity of the representation. In particular there is an explicit classification of those finite $p$-groups having a representation of fixity $f<p$.

Our main results are in the direction of propagating the natural free actions on $U(n) / U(k)$ to free actions on an actual product of spheres. For low fixity we can use explicit arguments involving equivariant vector bundles; in the case of fixity equal to one we have

Theorem 1.1. A subgroup $G \subset U(n)$ of fixity one acts freely and smoothly on $X=\mathbb{S}^{2 n-1} \times \mathbb{S}^{4 n-5}$. In particular if $G$ is any finite subgroup of $S U(3)$, then it will act freely and smoothly on $\mathbb{S}^{5} \times \mathbb{S}^{7}$.

From this we conclude that $A_{5}, S L_{3}\left(\mathbb{F}_{2}\right)$ and the triple cover $3 A_{6}$ all act freely and smoothly on $\mathbb{S}^{5} \times \mathbb{S}^{7}$.

Using the explicit classification of rank two $p$-groups (see [3]) as well as the description of $p$-groups of fixity equal to one in [24] we see that our result can be used to construct free actions for the exceptional (i.e. non-metacyclic) $p$-groups on the list. Combining this with the well-known fact that metacyclic groups act freely on a product of two linear spheres, we obtain

Theorem 1.2. Let $p>3$ be a prime. Then a p-group $P$ acts freely and smoothly on $\mathbb{S}^{m} \times \mathbb{S}^{n}$ for some $m, n>0$ if and only if $P$ does not contain $\mathbb{Z} / p \times \mathbb{Z} / p \times \mathbb{Z} / p$ as a subgroup.

This geometric result improves on the homotopy theoretic version recently established in [2]. Indeed this paper arose out of efforts to promote the results there to actions on manifolds. Moreover we obtain all of these actions explicitly, thus providing rank 2 models for group actions which may play a special role analogous to that of linear spheres. In contrast, the situation at the primes $p=2,3$ remains unresolved, reflecting the complications in the corresponding group theory at these primes. Recently Ünlü [31] has shown that among the 396 groups of order dividing 256 and which have rank equal to two, there is exactly one group which is not known to act freely and smoothly on a product of two spheres!

Using the methods developed in [2] we also obtain interesting results for the case of fixity equal to two, namely

Theorem 1.3. If $G \subset U(n)$ is of fixity equal to two, then $G$ acts freely on a finite complex $X \simeq \mathbb{S}^{2 n-1} \times \mathbb{S}^{4 n-5} \times \mathbb{S}^{M}$ for some $M>0$. In particular if $G \subset S U(4)$, then $G$ acts freely on a finite complex $X \simeq \mathbb{S}^{7} \times \mathbb{S}^{11} \times \mathbb{S}^{M}$ for some $M>0$.

For example, this can be used to show that $S p_{4}\left(\mathbb{F}_{3}\right)$ acts freely on a finite complex $X \simeq \mathbb{S}^{7} \times \mathbb{S}^{11} \times \mathbb{S}^{M}$ for some $M>0$.

For arbitrary fixity we must make use of methods from propagation theory, 
involving homotopy theory and surgery. Observe that the Stiefel manifolds $U(n) / U(n-f-1)$ have the cohomology of a product of $f+1$ spheres (see 4.1); our goal is to propagate this natural model to an action on a product of spheres. The main result in this paper is the following

Theorem 1.4. Let $G$ denote either a finite subgroup of $S U(n)$ and let $k=1$, or let $G$ denote a finite subgroup of $U(n)$ which acts freely on $U(n) / U(k)$ with $k \geq 1$. If the order of $G$ is prime to $(n-1)$ !, then $G$ acts freely, smoothly and homologically trivially on $\mathbb{S}^{2 n-1} \times \mathbb{S}^{2 n-3} \times \cdots \times \mathbb{S}^{2 k+1}$.

If $G \subset U(n)$ and $(|G|,(n-1) !)=1$, our methods yield a free $G$-action on a finite complex $X \simeq \mathbb{S}^{2 n-1} \times \cdots \times \mathbb{S}^{3} \times \mathbb{S}^{M}$ for some $M>1$; we conjecture that an analogue of our main theorem should also hold in this case, but there are surgery-theoretic difficulties to overcome which we hope to address in a subsequent paper.

Applying the available characterization of low fixity $p$-groups, we obtain interesting examples of group actions:

Theorem 1.5. Let $P$ denote a finite non-abelian p-group with cyclic center and having an abelian maximal subgroup. If the rank of $P$ is $r<p$, then there exists a free and homologically trivial action of $P$ on $M=\mathbb{S}^{2 p-1} \times \mathbb{S}^{2 p-3} \times \cdots \times \mathbb{S}^{2(p-r)+1}$, a product of $r$ spheres.

We should point out that this produces an infinite number of new examples of free actions by rank $r$ groups on a product of $r$ spheres. More generally it is conjectured that a rank $r$ finite group will act freely on a finite complex $X$ having the homotopy type of a product of $r$ spheres. A related conjecture is that every finite group acts freely and homologically trivially on a product of spheres. Although the condition $(|G|,(n-1) !)=1$ is somewhat restrictive, our approach does yield a new method for approaching the conjectures mentioned above; most importantly we have constructed many interesting geometric actions.

We are grateful to R. L. Griess for providing information concerning the classification of finite linear groups.

\section{Basic definitions and properties}

In this section we will recall the notion of fixity for a complex representation of a finite group $G$ (see [24]) and relate it to properties of associated actions on complex Stiefel manifolds $U(n) / U(k)$.

First we introduce some notation. Let $V$ denote a finite dimensional $\mathbb{C} G$ module. For a subgroup $H \subset G$, we denote by $V^{H}$ the subspace of vectors in $V$ fixed by all $h \in H$. We denote by $\langle g\rangle$ the subgroup generated by an element $g$ of $G$. 
Definition 2.1. The fixity of a finite dimensional $\mathbb{C} G$-module $V$ is

$$
\operatorname{Fix}_{G}(V)=\max _{1 \neq g \in G}\left\{\operatorname{dim}_{\mathbb{C}} \mathrm{V}^{\langle\mathrm{g}\rangle}\right\}
$$

Given a faithful complex representation $V$ of $G$, we can always obtain an equivalent unitary representation. Hence in what follows we will restrict our attention to faithful unitary representations and the associated embeddings $G \hookrightarrow U(n)$, where $U(n)$ denotes the group of $n \times n$ unitary matrices and $n=\operatorname{dim} V$. Given such an embedding and a closed subgroup $H \subset U(n)$, we have a natural $G$-action on the homogeneous space $U(n) / H$. In particular we will be interested in the subgroups $U(k) \subset U(n)$, defined by

$$
A \mapsto\left(\begin{array}{cc}
A & 0 \\
0 & I_{n-k}
\end{array}\right)
$$

where $I_{n-k}$ is the $(n-k) \times(n-k)$ identity matrix.

Note that $\operatorname{Fix}_{G}(V)$ can be expressed as the maximum (for $g \in G$ ) of the dimensions of the eigenspaces $\operatorname{ker}(g-I)$ for $g \neq 1$.

Lemma 2.2. $\operatorname{Fix}_{G}(V) \leq f$ if and only if the induced action of $G$ on $U(n) / U(n-f-1)$ is a free action.

Proof. If $\operatorname{Fix}_{G}(V) \leq f$, then given any $1 \neq g \in G$, $\operatorname{ker}(g-I)$ can be at most $f$-dimensional, hence $g$ cannot be conjugated into the subgroup $U(n-f-1)$ and so the action of $G$ on the homogeneous space $U(n) / U(n-f-1)$ must be free. Conversely if the action is free, no element can be conjugated into $U(n-f-1)$, hence $\operatorname{Fix}_{G}(V) \leq f$.

Corollary 2.3. A faithful $\mathbb{C} G$-module $V$ has fixity $f$ if and only if $f$ is the smallest integer such that the induced $G$-action on $U(n) / U(n-f-1)$ is free.

We can now reformulate the notion of fixity.

Definition 2.4. A faithful unitary representation $G \subset U(n)$ has fixity $f$ if $f$ is the smallest integer so that the induced action of $G$ on $U(n) / U(n-f-1)$ is free.

From this we derive an invariant associated to $G$.

Definition 2.5. For a finite group $G$, we define its fixity, $\operatorname{Fix}(G)$, as the minimum value of $\operatorname{Fix}_{G}(V)$, as $V$ ranges over all faithful, finite dimensional complex representations of $G$.

The case of fixity zero coincides with that of linear space forms. Indeed if an $n$-dimensional representation $V$ has fixity zero then the induced action on $U(n) / U(n-1)$ is free; this can be identified with the action on the sphere $S(V)$ 
of unit vectors in $V$. Examples of higher fixity are less well-known from the point of view of transformation groups, and they will provide building blocks for new examples of group actions.

Proposition 2.6. Let $G \subset S U(n)$. Then $G$ acts freely on $U(n) / U(1)$. Hence the fixity of $G$ is at most $n-2$.

Proof. Note det : $U(1) \stackrel{\simeq}{\rightarrow} S^{1}$. If $g \in G$ has a fixed point $h U(1) \in U(n) / U(1)$, then $h^{-1} g h \in U(1)$. Since $h^{-1} g h$ has determinant one, $h^{-1} g h=1$ so $g=1$.

We now relate fixity to another group-theoretic invariant.

Definition 2.7. For a finite group $G$ and a prime $p$, we define its $p$-rank as $r_{p}(G)=\max \left\{r \mid(\mathbb{Z} / p)^{r} \subset G\right\}$ and its rank as $r(G)=\max \left\{r_{p}(G) \mid p\right.$ divides $\left.|\mathrm{G}|\right\}$.

The following is a basic result relating rank to fixity ([28], Lemma 3.1):

Proposition 2.8. For any finite group $G, r(G) \leq \operatorname{Fix}(G)+1$.

Note that this inequality may be strict. For example, if $G$ is a non-abelian split extension of $\mathbb{Z} / p$ by $\mathbb{Z} / q$ where $p$ and $q$ are prime, then this is a rank one group which does not have a fixed point free representation (i.e. $G$ is not a Frobenius complement, as it does not satisfy the $p q$ condition); hence its fixity is greater than zero. For us the most interesting case occurs when $r(G)=\operatorname{Fix}(G)+1$.

Assume that $G \subset U(n)$ has fixity equal to $f$. Then the $G$ action on $U(n) / U(n-f)$ is not free. In fact we have

Proposition 2.9. If $G \subset U(n)$ has fixity $f$, then all of the isotropy subgroups for the $G$-action on $U(n) / U(n-f)$ have rank equal to one.

Proof. Let $H$ denote an isotropy subgroup for the $G$-action on $U(n) / U(n-f)$; this means that there exists a $g \in U(n)$ such that $g^{-1} H g \subset U(n-f)$. This subgroup then acts freely on the quotient $U(n-f) / U(n-f-1) \cong \mathbb{S}^{2(n-f)-1}$ as otherwise a conjugate of $G$ would intersect $U(n-f-1)$ non-trivially. Hence $H$ must be a group of rank equal to one.

Remark 2.10. More generally if we let $M_{k}=U(n) / U(k)$, this defines a sequence of $G$-manifolds $\left\{M_{0}, M_{1}, \ldots, M_{n}\right\}$ and equivariant maps $M_{k} \rightarrow M_{k+1}$, for $k=$ $0, \ldots, n$ where $M_{0}$ is a free $G$-space, $M_{n-1}=\mathbb{S}^{2 n-1}$ and $M_{n}=\left\{x_{0}\right\}$. As we go up this tower, the isotropy must increase from rank zero to rank $r(G)$. Using an argument similar to the one above, we see that the rank can only increase by one at each stage. There are $n$ steps and a total increase by $r(G)$ must happen. Hence we have a partition of $[0, n] \cap \mathbb{Z}$, given by integers $0 \leq s_{0}<s_{1}<\cdots<s_{r(G)} \leq n$ such that the isotropy of $M_{k}$ has rank $t$ if $s_{t} \leq k<s_{t+1}$. The patterns which arise 
in this process seem to be an interesting invariant of the representation; we shall make use of this in our applications. Note that when $n=r(G)$, the rank must increase exactly by one at every step; this is the case of maximal fixity $n-1$.

We now describe a characterization of $p$-groups of fixity $f<p$, which appears in [24].

Theorem 2.11. Let $f$ be a non-negative integer and let $p$ be a prime number greater than $f$. A non-abelian p-group $P$ has fixity $f$ if and only if the following hold:

- $P$ has p-rank equal to $f+1$;

- $P$ has cyclic center and an abelian maximal subgroup.

Moreover, for any such group there exists a faithful irreducible P-module of fixity $f$ and dimension $p$.

The $p$-groups of fixity $f<p$ have been explicitly described by Conlon in [9], and enumerated in [24]. Using this one can verify for example that for $p \geq 5$ and $n \geq 4$, there are exactly four non-abelian $p$-groups of order $p^{n}$ and fixity one. They can be listed as follows in terms of generators and relations (see [24], page 228 and [18], page 343):

- $<a, b \mid a^{p^{n-1}}=b^{p}=1, a^{b}=a^{1+p^{n-2}}>$

- $<a, x, y \mid a^{p^{n-3}}=[x, y], a^{p^{n-2}}=[a, x]=[a, y]=x^{p}=y^{p}=1>$

- $<a, x \mid a^{-\lambda p^{n-3}}=[x, a, x], a^{p^{n-2}}=x^{p}=[x, a]^{p}=[x, a, a]=1>$, where $\lambda$ is equal to 1 or to a non-quadratic residue modulo $p$.

Later we will construct actions of these $p$-groups on a product of two spheres. Note that the first group on this list is a metacyclic group; the other three are said to be of exceptional type and they appear in the classification of rank two $p$-groups which we will make use of in a subsequent section. The situation for $p=2$ is rather different: there are $3 n-8$ nonabelian groups of order $2^{n}$ having fixity equal to one.

In [28] a detailed analysis of group theoretic properties of groups of low fixity is carried out. The main result is that there exists a function $\theta: \mathbb{N} \rightarrow \mathbb{N}$ such that if $V$ is a $\mathbb{C} G$-module of fixity $f$, then there exists a normal subgroup $N \triangleleft G$ such that $[G: N] \leq \theta(f)$ and $N$ is solvable of derived length at most 3. Another result is that there exists a function $\Phi: \mathbb{N} \rightarrow \mathbb{N}$ such that if $V$ is a $\mathbb{C} G$-module of fixity $f$ and $\operatorname{dim} V>\Phi(f)$, then either $G$ is solvable or $G / O(G)$ has a subgroup of index at most 2 which is isomorphic to $\operatorname{SL}(2,5)$. Here $O(G)$ denotes the largest normal subgroup of $G$ whose order is odd, hence it is solvable.

There are substantial restrictions for groups of low fixity. For example, if $G$ is a finite group acting on $V$ with fixity equal to one, then we have

- $\operatorname{dim} V \leq|G|$. This is in contrast to the situation for fixity zero, where we can take modules of arbitrarily large dimension. In fact this is the only fixity where the modules do not have bounded dimension. 
- The $p$-rank of $G$ is one for all primes $p<\operatorname{dim} V-1$. For example this means that as soon as $\operatorname{dim} V>3$, the 2-Sylow subgroup of $G$ must be either cyclic or generalized quaternion. This excludes all non-abelian simple groups.

- If $p=2,3$ do not divide $|G|$, then there exists at most one prime $p$ with the property that $G$ has a non-abelian Sylow $p$-subgroup.

As we shall see later, groups of fixity one constitute a class of non-periodic groups that act freely and smoothly on a product of two spheres, and their structure may shed some light on the general conjecture that any finite group of rank equal to two acts freely on a product of two spheres. In this paper we prove this conjecture for $p$-groups with $p>3$.

\section{Actions of groups of fixity one and two}

In this section we begin by discussing the special case of groups having fixity equal to one. As we have seen they will act freely on some $U(n) / U(n-2)$, which happens to be a rather nice manifold which we can approach using very direct methods.

Theorem 3.1. A subgroup $G \subset U(n)$ of fixity one acts freely and smoothly on $X=\mathbb{S}^{2 n-1} \times \mathbb{S}^{4 n-5}$.

Proof. We have a fiber bundle

$$
\mathbb{S}^{2 n-3}=U(n-1) / U(n-2) \rightarrow U(n) / U(n-2) \rightarrow U(n) / U(n-1)=\mathbb{S}^{2 n-1},
$$

with structure group $U(n-1)$. It can be identified with the sphere bundle of an associated $(n-1)$-dimensional complex vector bundle $\xi$. In the next section we will see that the vector bundle $\xi$ is always non-trivial, but here we will note that $\xi \oplus \xi$ is trivial since it is classified by an element of $\pi_{2 n-1}(B U(2 n-2)) \cong$ $\pi_{2 n-2}(U(2 n-2)) \cong \pi_{2 n-2}(U)$, which is zero by Bott periodicity.

Note that $\xi$ is a $G$-vector bundle, and $G$ acts freely away from the zero section. By considering the pullback diagram

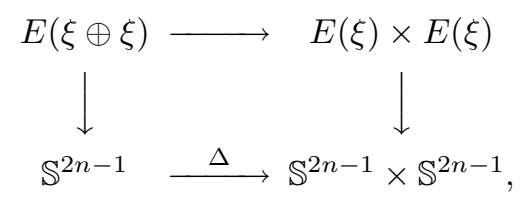

we see that $\xi \oplus \xi$ is also a $G$-vector bundle with action free away from the zero section. We can take $X=S(\xi \oplus \xi)$ (the associated sphere bundle) to complete the proof.

Corollary 3.2. Any finite subgroup of $S U(3)$ acts freely and smoothly on $\mathbb{S}^{5} \times \mathbb{S}^{7}$.

Example 3.3. The finite linear groups (to be precise we are referring to primitive unimodular irreducible groups) in low dimensions have been completely classified 
and listed by Blichtfeldt and others (see [4], [15]). For a fixed prime $p$ let $H_{p}$ denote the semidirect product of the form $E_{p} \times_{T} \mathrm{SL}_{2}\left(\mathbb{F}_{\mathrm{p}}\right)$, where

$$
E_{p}=\left\langle a, b, c \mid a^{p}=b^{p}=c^{p}=1,[a, b]=c\right\rangle
$$

is the extraspecial $p$-group of order $p^{3}$ and exponent $p$. Note that $\left|H_{p}\right|=$ $p^{4}(p-1)(p+1)$. We find four interesting subgroups of $S U(3)$ : the alternating group $A_{5}$, the simple linear group $S L_{3}\left(\mathbb{F}_{2}\right) \cong P S L_{2}\left(\mathbb{F}_{7}\right)$, the triple cover $3 A_{6}$, and $\mathrm{H}_{3}$. We obtain

Corollary 3.4. The groups $A_{5}, S L_{3}\left(\mathbb{F}_{2}\right), 3 A_{6}$, and $H_{3}$ all act freely and smoothly on $\mathbb{S}^{5} \times \mathbb{S}^{7}$.

Example 3.5. In [28] page 290, a group $G_{p} \subset U(p)$ of fixity equal to one and having order $2 p^{3}(p-1)$ is explicitly described as follows. Let $v_{0}, v_{1}, \ldots, v_{p-1}$ denote a basis for the underlying complex vector space and let $\omega$ denote a primitive $p$-th root of unity. Take the two linear transformations $A, B \in U(p)$ defined by $A v_{i}=\omega v_{i}$ and $B v_{i}=\omega^{i} v_{i}$ where $0 \leq i \leq p-1$. Now identify the set $\{0,1, \ldots, p-1\}$ with $\mathbb{F}_{p}$, and let $x$ be a generator of $\mathbb{F}_{p}^{*}$. Let $\mu \in \mathbb{C}$ be a primitive $2(p-1)$-th root of unity. We define $C, D \in U(p)$ via $C v_{i}=v_{i+1}, D v_{i}=\mu v_{x i}$, where $i \in \mathbb{F}_{p}$. Let $G_{p}$ denote the subgroup of $U(p)$ generated by $A, B, C, D$. The subgroup $H=\langle A, B\rangle \cong \mathbb{Z} / p \times \mathbb{Z} / p$ is normal in $G_{p}$, and $G_{p} / H$ is isomorphic to a double cover of the semi-direct product $\mathbb{Z} / p \times_{T} \mathbb{Z} /(p-1)$. The group $G_{p}$ acts freely and smoothly on $\mathbb{S}^{2 p-1} \times \mathbb{S}^{4 p-5}$.

These techniques are particularly effective for constructing actions of small p-groups.

Proposition 3.6. Let $P$ denote a p-group of rank equal to two and with order $|P| \leq p^{4}$. Then $P$ acts freely and smoothly on $\mathbb{S}^{m} \times \mathbb{S}^{n}$ for some $m, n>0$.

Proof. If the group $P$ has center $Z(P)$ of rank equal to two, then every element of order $p$ in $P$ is central. In this case the group acts freely on a product of two representations (see [2], page 422). Hence we can assume that $Z(P)$ is cyclic. For any $p$-group of order $p^{4}$, there exists an abelian subgroup of order $p^{3}$ (see [30], page 85), and so we can assume that $P$ has an abelian maximal subgroup. By Theorem 2.11 we infer that $P$ has fixity equal to one, whence by Theorem 3.1 it will act freely and smoothly on a product of two spheres, completing the proof.

As we have seen, if $G \subset U(n)$ has fixity one, then it will act on $U(n) / U(n-1)=$ $\mathbb{S}^{2 n-1}$ with rank one isotropy subgroups. In [2], it was shown that given a linear $G$-sphere $S(V)$ satisfying this condition, there exists a finite $G$-CW complex $X$ with a free $G$-action, and such that $X \simeq S(V) \times \mathbb{S}^{M}$ for some integer $M$ (see [31] for a more direct proof). From the above we see that for a group $G$ of fixity one, this construction can be realized explicitly via a free and smooth $G$-action 
on $\mathbb{S}^{4 n-5} \times \mathbb{S}^{2 n-1}$. However there are groups which act on a linear sphere with periodic isotropy which do not have fixity equal to one. Indeed in [2] it was shown that every rank two $p$-group admits a representation in $U(|P: Z(P)|)$ such that the action on $U(|P: Z(P)|) / U(|P: Z(P)|-1)$ has periodic isotropy; on the other hand we have seen that $p$-groups of fixity equal to one are rather restricted. However in the case of rank two $p$-groups we have complete information:

Proposition 3.7. If $p>3$ and $P$ is a finite p-group of rank equal to two, then $P$ is either a metacyclic group or a group of exceptional type, with fixity equal to one.

This proposition is proved in [24], page 228 by using the classification of rank two $p$-groups appearing in [3], as well as the characterization of $p$-groups of fixity equal to one described in Lemma 2.11. Next we recall the proof of an elementary

Lemma 3.8. If $P$ is a metacyclic p-group then it acts freely on a product of two linear spheres.

Proof. The group $P$ is an extension of the form $1 \rightarrow \mathbb{Z} / p^{t} \rightarrow P \rightarrow \mathbb{Z} / p^{s} \rightarrow 1$. Let $\chi$ denote a one dimensional character of the subgroup $B \cong \mathbb{Z} / p^{t}$ which maps a generator of $B$ to a primitive $p^{t}$-th root of unity. Let $V=\operatorname{Ind}_{B}^{P}(\chi)$; this is a $p^{s}$ dimensional complex representation of $P$. The action of $P$ on the associated sphere $S(V)$ restricts to a free $B$-action; this can be checked using Mackey's formula (it suffices to show that the unique and central subgroup of order $p$ in $B$ acts freely). Now let the quotient group $A=P / B$ act freely on $\mathbb{S}^{1}$ through multiplication by a primitive $p^{s}$-th root of unity. From this we can define a diagonal $P$ action on $\mathbb{S}^{2 p^{s}-1} \times \mathbb{S}^{1}$ which is evidently free.

Obviously the action described above is a product action. An interesting fact is that for $p>3$, any exceptional rank two $p$-group does not admit a free product action; indeed we have

Proposition 3.9. If $p>3$ and $P$ is a rank two p-group which is not metacyclic, then it cannot act freely on $X=\mathbb{S}^{m} \times \mathbb{S}^{n}$ via a product action.

Proof. By the results in [14], every action of a finite $p$-group on a sphere can be modeled using a linear action; in particular given such a product action there exist representations $V, W$ such that for any $g \in P$ we have $X^{\langle g\rangle} \neq \emptyset$ if and only if $V^{\langle g\rangle} \neq\{0\}$ and $W^{\langle g\rangle} \neq\{0\}$. In particular this implies that if $P$ acts freely on $X$, then it acts freely on $S(V) \times S(W)$. However it is shown in [26], page 486 that this is impossible for a non-metacyclic $p$-group if $p>3$.

We can now state and prove a geometric characterization of rank two $p$-groups (for $p>3$ ) which naturally extends the classical rank one situation, and which 
rather surprisingly has been a conjecture until now:

Theorem 3.10. Let $p>3$ be a prime number. Then a p-group $P$ acts freely and smoothly on $M=\mathbb{S}^{m} \times \mathbb{S}^{n}$ for some $m, n>0$ if and only if $P$ does not contain $\mathbb{Z} / p \times \mathbb{Z} / p \times \mathbb{Z} / p$ as a subgroup.

Proof. We have known for decades that $(\mathbb{Z} / p)^{3}$ does not act freely on a product of two spheres (see [16]). All we need to do is construct the actions. By Proposition 3.7, we know that $P$ is either metacyclic, or of exceptional type and having fixity equal to one. By Lemma 3.8, the metacyclic case is taken care of; by Theorems 2.11 and 3.1 , the rank two $p$-groups of exceptional type will all act freely and smoothly on $\mathbb{S}^{2 p-1} \times \mathbb{S}^{4 p-5}$.

The situation at the 'small primes' $p=2$ and $p=3$ is still unresolved. It can be shown that for $n>4$, there exist exactly two 3 -groups of order $3^{n}$ which are neither of fixity one, nor act freely on a product of two linear spheres. At the prime 2 the group theory is considerably more complicated. We briefly summarize recent work by $\ddot{O}$. Ünlü in this direction (see [31]). The smallest example of a rank two 2-group of fixity greater than one that does not act freely on a product of two linear spheres is the extraspecial 2-group $Q_{8} * D_{8}=Q_{8} \times D_{8} / \Delta$, where $\Delta$ is the diagonal central subgroup of order 2 . Note that $Q_{8} * D_{8}$ is of order 32 and does not contain a maximal subgroup which is abelian. In fact one can show that a rank two 2-group $P$ does not contain $Q_{8} * D_{8}$ if and only if it either has fixity equal to one or acts freely on a product of two linear spheres. Hence all such groups act freely and smoothly on a product of two spheres. In order to handle this particular group, we need the notion of quaternionic fixity, expressed in terms of representations $G \subset S p(n)$. In the case of $Q_{8} * D_{8}$, it embeds in $S p(2)$, and twice the associated bundle is seen to split, hence producing a free and smooth action of this group on $\mathbb{S}^{7} \times \mathbb{S}^{7}$. Using these techniques, Ünlü has shown that among the 396 groups of rank equal to two, and having order which divides 256 , there is exactly one group which is not known to act freely and smoothly on a product of two spheres. Verifying this requires using the computer algebra program MAGMA.

Remark 3.11. It seems worthwhile to compare our results with those obtained in [20] and [35] for free $p$-group actions on a product of two equidimensional spheres. From their work we know for example that the extraspecial $p$-group of order $p^{3}$ and exponent $p$ ( $p$ an odd prime) cannot act freely on any $\mathbb{S}^{n} \times \mathbb{S}^{n}$. An interesting related problem is determining general restrictions on $n$ and $m$ imposed by the existence of a free $P$-action on $\mathbb{S}^{n} \times \mathbb{S}^{m}$. Our construction produces a homogeneous system of parameters $\{\alpha, \beta\}$ for $H^{*}\left(P, \mathbb{F}_{p}\right)$ such that $|\alpha|=n+1$ and $|\beta|=m+1$ (see [2], page 419). This is a significant cohomological restriction, which deserves further consideration. 
For fixity equal to two we can apply the results in [2] to obtain a free action on a finite complex, although not necessarily a manifold. As a consequence we will obtain new examples of free actions for rank three groups.

Theorem 3.12. If $G \subset U(n)$ is of fixity equal to two, then $G$ acts freely on a finite complex $X \simeq \mathbb{S}^{2 n-1} \times \mathbb{S}^{4 n-5} \times \mathbb{S}^{M}$ for some $M>0$.

Proof. Consider the $G$-action on $Y=U(n) / U(n-2)$; by our hypothesis it has rank one isotropy. Using the same approach as in Theorem 3.1 we obtain a $G$-action on $Y^{\prime}=\mathbb{S}^{2 n-1} \times \mathbb{S}^{4 n-5}$ with the same isotropy, hence of rank equal to one. Applying 1.4 in [2] we obtain that $G$ acts freely on a finite complex $X \simeq Y^{\prime} \times \mathbb{S}^{M}$ for some $M>0$, which completes the proof.

Corollary 3.13. If $G \subset S U(4)$ then $G$ acts freely on a finite complex $X \simeq \mathbb{S}^{7} \times$ $\mathbb{S}^{11} \times \mathbb{S}^{M}$ for some $M>0$.

Proof. It suffices to observe that if $G \subset S U(4)$, then it will act on $S U(4) / S U(2)$ with isotropy of rank at most one.

Example 3.14. We are of course interested in groups of rank equal to three and of fixity equal to two. The list of finite linear subgroups in [15] yields two interesting examples in $S U(4)$. Let $T=\mathrm{SL}_{2}\left(\mathbb{F}_{3}\right) * \mathrm{SL}_{2}\left(\mathbb{F}_{3}\right)=\mathrm{SL}_{2}\left(\mathbb{F}_{3}\right) \times \mathrm{SL}_{2}\left(\mathbb{F}_{3}\right) / \Delta$, where $\Delta \cong \mathbb{Z} / 2$ is the diagonal subgroup of order two. Note that $|T|=2^{5} \cdot 3^{2}$ and that its 2-Sylow subgroup is the central product $Q_{8} * Q_{8}$, which is an extra-special 2-group of order 32 and has rank equal to three. The second example is $G=\operatorname{Sp}_{4}\left(\mathbb{F}_{3}\right)$, in this case $|G|=2^{6} \cdot 3^{4} \cdot 5$. It has 2 -rank and 3 -rank both equal to three. Hence we obtain

Corollary 3.15. The groups $\mathrm{SL}_{2}\left(\mathbb{F}_{3}\right) * \mathrm{SL}_{2}\left(\mathbb{F}_{3}\right)$ and $\mathrm{Sp}_{4}\left(\mathbb{F}_{3}\right)$ act freely on a finite complex of the form $X \simeq \mathbb{S}^{7} \times \mathbb{S}^{11} \times \mathbb{S}^{M}$ for some $M>0$.

Example 3.16. From the Atlas [10] we see that there is an embedding of $G=$ $\mathrm{PSL}_{2}\left(\mathbb{F}_{8}\right)$ in $S U(7)$. Note that $|G|=2^{3} \cdot 3^{2} \cdot 7$ and that the 3 -Sylow subgroup is cyclic while the 2-Sylow subgroup $S$ is elementary abelian. From the character table in the Atlas we can infer that this 7-dimensional representation $V$ restricts to the reduced regular representation on the subgroup $S$. Hence on any rank two subgroup $E \subset S, V_{\left.\right|_{E}} \cong I \oplus I \oplus \mathbb{C}$, where $I$ is the 3 -dimensional reduced regular representation. This representation clearly cannot be conjugated into the subgroup $S U(5) \subset S U(7)$ defined as before by extending matrices in $S U(5)$ by the $2 \times 2$ identity matrix. Hence $E$ acts with cyclic isotropy on $S U(7) / S U(5)$. Applying the techniques outlined above, we infer that $G=\mathrm{PSL}_{2}\left(\mathbb{F}_{8}\right)$ acts freely on a finite complex $X \simeq \mathbb{S}^{13} \times \mathbb{S}^{23} \times \mathbb{S}^{M}$ for some $M>0$. Note that in this example $G$ acts freely on $S U(7) / S U(3)$ but not on $S U(7) / S U(4)$, hence $G \subset S U(7)$ has fixity equal to three; nevertheless our methods can be applied to this representation. 
More generally if $G$ acts on $U(n) / U(n-f)$ with periodic isotropy, the results in [2] establish the existence of a free action on a finite complex $X \simeq U(n) / U(n-f)$ $\times \mathbb{S}^{M}$ for some large $M$.

In the next section we use surgery theory (propagation of group actions) to construct a free action of a subgroup $G \subset U(n)$ of fixity $f$ on a product of $f+1$ spheres provided the order of $G$ is prime to $(n-1)$ !. If $G$ is such a group and has fixity one, these techniques provide a stronger result than Theorem 3.1 in the sense that the action is on a lower-dimensional product of spheres.

\section{Propagating group actions}

In this section we will show that a free action of a group of order prime to $(n-1)$ ! on a Stiefel manifold $U(n) / U(k)$ propagates to a free action on a product of spheres. To begin we recall the integral cohomology of Stiefel manifolds.

Proposition 4.1. The Stiefel manifold $U(n) / U(k)$ has the same integral cohomology as a product $\mathbb{S}^{2 n-1} \times \mathbb{S}^{2 n-3} \times \cdots \times \mathbb{S}^{2 k+1}$ of $n-k$ spheres.

Proof. We will prove this by downward induction on $k$; clearly it is true for $k$ equal to $n-1$. Consider the fibration $\mathbb{S}^{2 k+1} \rightarrow U(n) / U(k) \rightarrow U(n) / U(k+1)$; the Euler class must be zero since, using the inductive hypothesis, it sits in a zero group. Hence the associated Gysin sequence breaks into a sequence of short exact sequences. We infer that the cohomology of $U(n) / U(k)$ is an exterior algebra on generators in the desired dimensions.

One would not expect the Stiefel manifold to have the same homotopy type as a product of spheres, since Bott periodicity forces a regularity on the homotopy groups of Stiefel manifolds which is absent from the homotopy groups of a product of spheres. However, the differences only involve primes less than $n$. The next several results illustrate these phenomena.

Proposition 4.2. $\pi_{2 n}(U(n)) \cong \mathbb{Z} / n$ ! and the generator is given by the characteristic element of the fibration $U(n) \rightarrow U(n+1) \rightarrow S^{2 n+1}$.

Proof. This result is due to Bott [5], however we will sketch a proof which uses only stable Bott periodicity. Note that $\pi_{2 n}(U(n+1))=\pi_{2 n}(U)=0$ and $\pi_{2 n+1}(U(n+1))$ $=\pi_{2 n+1}(U)=\mathbb{Z}$ (these groups are in the stable range), hence we have an exact sequence

$$
\pi_{2 n+1}(U(n+1)) \stackrel{p_{*}}{\longrightarrow} \pi_{2 n+1}\left(\mathbb{S}^{2 n+1}\right) \stackrel{\Delta_{*}}{\longrightarrow} \pi_{2 n}(U(n)) \rightarrow 0 .
$$

Recall (see [34], page 206) that the characteristic element of the fibration above is given by $\Delta_{*}\left(\iota_{2 n+1}\right) \in \pi_{2 n}(U(n))$, where $\iota_{2 n+1}$ is the canonical generator for $\pi_{2 n+1}\left(\mathbb{S}^{2 n+1}\right)$. Hence $\pi_{2 n}(U(n))$ is cyclic with generator the characteristic element. 
The homomorphism $p_{*}$ can be identified with taking the Euler class of the corresponding $(n+1)$-dimensional complex bundle over $\mathbb{S}^{2 n+2}$, and hence with the top Chern class $c_{n+1}$. But the Chern classes which arise from bundles over $\mathbb{S}^{2 n+2}$ are precisely the multiples of $n$ ! (see [19], page 280). The result follows.

The following technical proposition will be a key ingredient in our construction of group actions.

Proposition 4.3. Given integers $n>k$, there is a map

$$
\mathbb{S}^{2 n-1} \times \mathbb{S}^{2 n-3} \times \cdots \times \mathbb{S}^{2 k+1} \rightarrow U(n) / U(k)
$$

which induces an isomorphism in homology with coefficients in $\mathbb{Z}\left[\frac{1}{(n-1) !}\right]$.

Proof. Consider the fiber bundle

$$
U(n-1) / U(k) \rightarrow U(n) / U(k) \rightarrow \mathbb{S}^{2 n-1}
$$

with structure group $U(n-1)$. By Proposition 4.1 (or a Wang sequence)

$$
H^{*}(U(n) / U(k) ; \mathbb{Z})=H^{*}\left(\mathbb{S}^{2 n-1} ; \mathbb{Z}\right) \otimes H^{*}(U(n-1) / U(k) ; \mathbb{Z}) .
$$

By Proposition 4.2 the fiber bundle is classified by the homotopy class $\mathbb{S}^{2 n-1} \rightarrow$ $B U(n-1)$ representing the generator of $\pi_{2 n-1}(B U(n-1))=\mathbb{Z} /(n-1)$ !. Hence if we take a map $g: \mathbb{S}^{2 n-1} \rightarrow \mathbb{S}^{2 n-1}$ of degree $(n-1)$ ! then the induced bundle with fiber $U(n-1) / U(k)$ will be trivial, with total space $E$ homeomorphic to $\mathbb{S}^{2 n-1} \times U(n-1) / U(k)$; note that it comes equipped with a map $E \rightarrow U(n) / U(k)$ which induces a $\mathbb{Z}\left[\frac{1}{(n-1) !}\right]$ homology equivalence. Using downward induction on $n$ we can easily obtain the desired map.

Hence the Stiefel manifold $U(n) / U(k)$ has the same integral cohomology as a product of spheres, and after inverting $(n-1)$ !, we can realize this isomorphism by a map from the product of spheres to the Stiefel manifold. Under these conditions, if we have a free action of a group on $U(n) / U(k)$ and if the order of the group is prime to $(n-1)$ !, then the action will "propagate" to a free action on an actual product of spheres.

Theorem 4.4. Let $G$ denote a finite subgroup of $U(n)$ which acts freely on $U(n) / U(k)$ with $k \geq 1$. If the order of $G$ is prime to $(n-1)$ !, then $G$ acts freely and smoothly on $\mathbb{S}^{2 n-1} \times \mathbb{S}^{2 n-3} \times \cdots \times \mathbb{S}^{2 k+1}$.

Remark 4.5. In the proof of the theorem we use the fact that $U(n) / U(k)$ is simply-connected when $k \geq 1$. It seems likely that Theorem 4.4 is true in the nonsimply-connected case $G \subset U(n)(k=0)$, but the surgery theoretic complications are considerable, and we will not consider them here. However our methods will still allow us to construct a free $G$-action on a finite complex with the homotopy type of a product of $n$ spheres (see Corollary 4.13). 
The proof of Theorem 4.4 uses the method of propagation of group actions. We refer to [8] for the basic technique, to [1] for a short survey, to [12] for a nice application, to [11] for a key lemma, and to [13] for definitive statements of results. The philosophy is that if two manifolds (e.g. the Stiefel manifold and the product of spheres) resemble each other homologically at the order of a finite group $G$, then their behavior with respect to $G$-actions should be similar. To get actions on manifolds, the methods are surgery theoretic and the technical details can be formidable.

Definition 4.6. A $G$-action on $Y$ propagates across a map $f: X \rightarrow Y$ if there is a $G$-action on $X$ and an equivariant map homotopic to $f$.

Definition 4.7. A $G$-action on a space $X$ is homologically trivial if the induced action on $H_{*}(X ; \mathbb{Z}[1 / q])$ is trivial, where $q$ is the order of $G$.

The main theorem involving propagation is stated below. We will spend the rest of the section defining terms in its statement, outlining the proof, and applying it to the case of interest (Theorem 4.4). Most of the theorem below is due to Cappell-Weinberger [8], however the general case is due to Davis-Löffler [11]. We outline the proof since that will make application of the theorem easier, and also because the full statement is not easy to find in the literature.

Theorem 4.8. Let $f: X \rightarrow Y$ be a map between simply-connected spaces having the homotopy type of $C W$-complexes. Let $G$ be a group of order $q$ acting freely and homologically trivially on $Y$. Consider the following conditions:

(1) $f$ is a $\mathbb{Z}_{(q)}$-equivalence:

$$
f_{*}: H_{*}\left(X ; \mathbb{Z}_{(q)}\right) \stackrel{\simeq}{\longrightarrow} H_{*}\left(Y ; \mathbb{Z}_{(q)}\right) .
$$

(2) The Swan obstruction vanishes:

$$
\sigma\left(\chi^{\text {tor }}(f)\right)=0 \in \widetilde{K}_{0}(\mathbb{Z} G) .
$$

(3) $X$ and $Y$ are closed smooth manifolds of dimension greater than four and the action of $G$ on $Y$ is smooth.

(4) The normal invariant of the $\mathbb{Z}_{(q)}$-local homotopy equivalence $f$ is in the image of the transfer map:

$$
\nu_{(q)}(f) \in \operatorname{im}\left(p^{*}:[Y / G, F / O]_{(q)} \rightarrow[Y, F / O]_{(q)}\right) .
$$

Then

(a) If (1) holds, there is a $C W$-complex $X^{\prime}$ and a homotopy equivalence $h$ : $X^{\prime} \rightarrow X$ so that the $G$-action on $Y$ propagates across $f \circ h$, with a cellular $G$ action on $X^{\prime}$. Furthermore, the homotopy type of $X^{\prime} / G$ is uniquely determined.

(b) If (1) holds and $Y / G$ and $X$ have the homotopy type of finite-dimensional $C W$-complexes, there is a finite-dimensional $C W$-complex $X^{\prime}$ satisfying the conclusion of (a). 
(c) If (1) and (2) hold and $Y / G$ and $X$ have the homotopy type of finite $C W$ complexes, there is a finite $C W$-complex $X^{\prime}$ satisfying the conclusion of (a).

(d) If $q$ is odd and (1), (2) and (3) hold, then $X^{\prime}$ can be taken to be a closed smooth manifold.

(e) If $q$ is odd and (1), (2), (3) and (4) hold, then the $G$-action on $Y$ propagates across $f$, with a smooth action on $X$.

Before sketching a proof of Theorem 4.8 we recall some basic background material. The homotopy aspects of propagation of group actions depend on localizing topological spaces (see [17], [6], and [7] for details). Let $R \subset \mathbb{Q}$ be a subring of the rationals. For an abelian group $A$, let $A_{R}=A \otimes R$. A homomorphism $A \rightarrow B$ is an $R$-equivalence if the induced map $A_{R} \rightarrow B_{R}$ is an isomorphism. An abelian group $A$ is $R$-local if the map $A \rightarrow A_{R}$ is an isomorphism.

We assume any space discussed below has the homotopy type of a connected CW-complex. A map $X \rightarrow Y$ is an $R$-equivalence if the induced map on the fundamental group is an isomorphism and the induced maps on higher homotopy groups $\pi_{n}, n>1$ are $R$-local equivalences. Equivalently, the map is an isomorphism on the fundamental group and the induced map on the homology of the universal covers $H_{*}(\widetilde{X}) \rightarrow H_{*}(\widetilde{Y})$ is an $R$-equivalence. A space $X$ is $R$-local if $\pi_{n}(X)$ is $R$-local for all $n>1$, equivalently $H_{*}(\tilde{X})$ is $R$-local. An $R$-localization of $X$ is a $R$-equivalence $X \rightarrow Y$ where $Y$ is $R$-local. There are existence and uniqueness theorems for $R$-localizations of $X$. Their existence follows by applying the fiberwise localization theorem of Bousfield-Kan [7, p. 40] to $X \rightarrow B \pi_{1} X$. Their $R$-localization is functorial on the geometric realizations of simplicial sets and maps. If $f: X \rightarrow Y$ and $g: X \rightarrow Z$ are two $R$-localizations of $X$, then there is a homotopy equivalence $h: Y \rightarrow Z$ so that $h \circ f \simeq g$. We will write $X \rightarrow X_{R}$ to denote an $R$-localization of $X$.

Let $q$ be a nonzero integer. Let

$$
\mathbb{Z}_{(q)}=\mathbb{Z}\left[\frac{1}{p_{1}}, \frac{1}{p_{2}}, \frac{1}{p_{3}}, \ldots\right] \subset \mathbb{Q},
$$

where $\left\{p_{1}, p_{2}, p_{3}, \ldots\right\}$ is the set of primes which do not divide $q$. For a space $X$, let $X \rightarrow X_{(q)}$ and $X \rightarrow X[1 / q]$ denote the $R$-localizations of $X$ where $R$ equals $\mathbb{Z}_{(q)}$ and $\mathbb{Z}[1 / q]$ respectively. Then $X$ is the homotopy pullback of

$$
\begin{aligned}
& \quad \\
& X_{(q)} \longrightarrow X_{\mathbb{Q}}
\end{aligned}
$$

(i.e. $X$ is homotopy equivalent to what results after converting the vertical map to a fibration and taking the pullback).

S. Weinberger [33] made the following key observation.

Lemma 4.9. A free cellular $G$-action on a simply-connected $C W$-complex $X$ is 
homologically trivial if and only if $(X / G)[1 / q] \simeq X[1 / q] \times B G$.

Proof. Suppose a finite group $G$ of order $q$ acts freely on a simply-connected space $X$. Then $X / G \subset Y=(X / G) \cup e_{1}^{2} \cup \cdots \cup e_{k}^{2}$ with $Y$ simply-connected (add 2 -cells to kill the fundamental group). Since $H_{1}(X / G)=G /[G, G]$ is $q$-torsion, the map $\alpha$ below is an epimorphism:

$$
\pi_{2} Y \cong H_{2} Y \rightarrow H_{2}(Y ; \mathbb{Z}[1 / q]) \stackrel{\alpha}{\rightarrow} H_{2}(Y, X / G ; \mathbb{Z}[1 / q]) \cong \mathbb{Z}[1 / q]^{k} .
$$

Let $Z=Y \cup e_{1}^{3} \cup \cdots \cup e_{k}^{3}$ where the attaching maps of the 3-cells represent a $\mathbb{Z}[1 / q]$-basis of $H_{2}(Y, X / G ; \mathbb{Z}[1 / q])$. By means of this "plus" construction we have constructed a map $i: X / G \rightarrow Z$ to a simply-connected space inducing an isomorphism on $H_{*}(\quad ; \mathbb{Z}[1 / q])$.

Now suppose, in addition, that $G$ acts homologically trivially on $X$. Then a transfer argument shows that the covering map $\pi: X \rightarrow X / G$ induces an isomorphism on $H_{*}(; \mathbb{Z}[1 / q])$, hence so does $i \circ \pi: X \rightarrow Z$. Thus $Z[1 / q] \simeq$ $X[1 / q]$, so we have $\mathbb{Z}[1 / q]$-equivalence $X / G \rightarrow X[1 / q] \times B G$ to a $\mathbb{Z}[1 / q]$-space. By uniqueness of localization, there is a homotopy equivalence $(X / G)[1 / q] \rightarrow$ $X[1 / q] \times B G$ as desired.

Conversely if $(X / G)[1 / q] \simeq X[1 / q] \times B G$, then the $G$-action on $X$ is homologically trivial since the $G$-action on $X[1 / q] \times E G$ clearly is.

One could also prove the above lemma by using obstruction theory to show that the fibration

$$
X[1 / q] \rightarrow X[1 / q] \times_{G} E G \rightarrow B G
$$

is fiber homotopically trivial and noting $X[1 / q] \times_{G} E G \simeq X / G[1 / q]$.

Proof of Theorem 4.8 (a). Let $X^{\prime} / G$ be a CW-complex having the homotopy type of the homotopy pullback of

$$
\begin{aligned}
& X[1 / q] \times B G \\
& \downarrow \\
&(Y / G)_{(q)} \longrightarrow Y_{\mathbb{Q}} \times B G
\end{aligned}
$$

where the vertical map is given by applying $f[1 / q]$ and then $\mathbb{Q}$-localization, and the horizontal map is provided by applying $\mathbb{Q}$-localization and then Weinberger's Lemma.

Proof of Theorem 4.8 (b). We will use the criterion of Wall [32, Thm. E] which says that a CW-complex $Z$ has the homotopy type of an complex of dimension $N$ if and only if $H^{i}(\widetilde{Z} ; \mathbb{Z})=0$ for all $i>N$ and $H^{N+1}(Z ; M)=0$ for all local coefficient systems $M$.

Recall that both $Y / G$ and $X$ are assumed to have the homotopy type of finite dimensional $\mathrm{CW}$-complexes; let $N$ be greater than or equal to both dimensions. 
Let $X^{\prime} / G$ be a CW-complex produced by Theorem 4.8 (a). Then for $i>N$, $H^{i}\left(\widehat{X^{\prime} / G} ; \mathbb{Z}\right)=H^{i}(X ; \mathbb{Z})=0$. Let $M$ be a local coefficient system for $X^{\prime} / G$. For a prime $p$ dividing the order of $G, H^{N+1}\left(X^{\prime} / G ; M\right)_{(p)}$ is isomorphic to

$$
H^{N+1}\left(\left(X^{\prime} / G\right)_{(p)} ; M_{(p)}\right) \cong H^{N+1}\left((Y / G)_{(p)} ; M_{(p)}\right) \cong H^{N+1}(Y / G ; M)_{(p)}=0 .
$$

Likewise for a prime $p$ not dividing the order of $G$, a transfer argument shows

$$
\begin{aligned}
H^{N+1}\left(X^{\prime} / G ; M\right)_{(p)} & \cong H^{N+1}\left(\left(X^{\prime} / G\right)_{(p)} ; M_{(p)}\right) \\
& \cong H^{N+1}\left(X_{(p)}^{\prime} ; M_{(p)}\right) \cong H^{N+1}(X ; M)_{(p)}=0 .
\end{aligned}
$$

Hence $H^{N+1}\left(X^{\prime} / G ; M\right)=0$. Thus $X^{\prime} / G$ has the homotopy type of an $N$-complex.

This leads to a nontrivial result.

Corollary 4.10. Let $G$ denote a finite subgroup of $U(n)$ which acts freely on $U(n) / U(k)$ with $k \geq 1$. Suppose the order of $G$ is prime to $(n-1) !$. Then $G$ acts freely on a finite dimensional $C W$-complex having the homotopy type of $\mathbb{S}^{2 n-1} \times \mathbb{S}^{2 n-3} \times \cdots \times \mathbb{S}^{2 k+1}$.

It is traditional in the study of actions on products of spheres to be satisfied with an action on a finite-dimensional complex which has the homotopy type of a product of spheres. But that is not good enough for us; we won't stop until we have constructed an action on the product of spheres themselves! Next we will try and improve from a finite-dimensional complex to a finite complex.

Wall [32] showed that a connected CW-complex $W$ has the homotopy type of a finite CW-complex if and only if $\pi_{1}(W)$ is finitely presented and $C_{*}(\widetilde{W})$ is chain homotopy equivalent to finite free $\mathbb{Z}\left[\pi_{1} W\right]$-chain complex. If $C_{*}(\widetilde{W})$ is finitely dominated this is equivalent to the vanishing of the finiteness obstruction $\left[C_{*}(\widetilde{W})\right] \in \widetilde{K}_{0}\left(\mathbb{Z}\left[\pi_{1}(W)\right]\right)$.

We recall the definition of the Swan homomorphism appearing in Theorem 4.8. For an integer $r$ relatively prime to $q=|G|$, define the projective $\mathbb{Z} G$-module $P_{r}=$ $\operatorname{ker} \epsilon: \mathbb{Z} G \rightarrow \mathbb{Z} / r$. One can show that $P_{r} \oplus P_{s} \cong P_{r s} \oplus \mathbb{Z} G$ and that $P_{1+k q} \cong \mathbb{Z} G$. Hence there is a well-defined Swan homomorphism $\sigma:(\mathbb{Z} / q)^{\times} \rightarrow \widetilde{K}_{0}(\mathbb{Z} G)$, defined by $\sigma([r])=\left[P_{r}\right]$. Now given a rational homology equivalence $f: X \rightarrow Y$, define $\chi^{\text {tor }}(f)$ to be the rational number $\prod_{i}\left|H_{i}\left(C_{f}\right)\right|^{(-1)^{i}}$, where $C_{f}$ is the mapping cone of $f$. If $f$ is a $\mathbb{Z}_{(q)}$-equivalence, then $\chi^{\text {tor }}(f) \in \mathbb{Z}_{(q)}^{\times}$and hence determines an element $\left[\chi^{\text {tor }}(f)\right] \in(\mathbb{Z} / q)^{\times}$.

Proof of Theorem 4.8 (c). By Theorem 4.8 (a) there is a CW-complex $Z$, a homotopy equivalence $h_{Z}: Z \rightarrow X$, a free, cellular $G$-action on $Z$, and an equivariant map $f_{Z}: Z \rightarrow Y$ so that $f_{Z} \simeq f \circ h_{Z}$. There is a short exact sequence of chain 
complexes

$$
0 \rightarrow C_{*}(Y) \rightarrow C_{*}\left(C_{f_{Z}}\right) \rightarrow \Sigma C_{*}(Z) \rightarrow 0 .
$$

Our goal is to show that $C_{*}(Z)$ is finitely dominated and that the finiteness obstruction $\left[C_{*}(Z)\right] \in \widetilde{K}_{0}(\mathbb{Z} G)$ vanishes. Since $\oplus H_{i}\left(C_{f_{Z}}\right)$ is finitely generated and each $H_{i}\left(C_{f_{Z}}\right)$ has a finite resolution over $\mathbb{Z} G, C_{*}\left(C_{f_{Z}}\right)$ is finitely dominated, as is $C_{*}(Y)$. Hence $\Sigma C_{*}(Z)$ is finitely dominated and

$$
\left[C_{*}(Z)\right]=-\left[\Sigma C_{*}(Z)\right]=\left[C_{*}(Y)\right]-\left[C_{*}\left(C_{f_{Z}}\right)\right]=-\sigma\left(\left[\chi^{\text {tor }}(f)\right]\right) .
$$

Thus $C_{*}(Z)$ is chain homotopy equivalent to a finite free $\mathbb{Z} G$ chain complex (equivalently $Z / G$ has the homotopy type of a finite $\mathrm{CW}$-complex) if and only if $\sigma\left(\left[\chi^{\text {tor }}(f)\right]\right)=0$.

Corollary 4.11. Let $G$ be a subgroup of $U(n)$ which acts freely on $U(n) / U(k)$ with $k \geq 1$ and whose order is prime to $(n-1)$ !. Then $G$ acts freely on a finite $C W$-complex having the homotopy type of $\mathbb{S}^{2 n-1} \times \mathbb{S}^{2 n-3} \times \cdots \times \mathbb{S}^{2 k+1}$.

Proof. Here are two properties of $\chi^{\text {tor }}$, whose verification is left to the reader.

- $\chi^{\text {tor }}(f \circ g)=\chi^{\text {tor }}(f) \chi^{\text {tor }}(g)$.

- If $A_{*}, B_{*}$, and $C_{*}$ are graded abelian groups with $C_{*}$ free, and if $f_{*}: A_{*} \rightarrow B_{*}$ is a graded map, then $\chi^{\text {tor }}\left(f_{*} \otimes \operatorname{Id}_{C_{*}}\right)=\chi^{\text {tor }}\left(f_{*}\right)^{\chi\left(C_{*}\right)}$.

In the proof of Proposition 4.3 we constructed a $\mathbb{Z}_{(q)}$-equivalence

$$
f_{n, k}: \mathbb{S}^{2 n-1} \times U(n-1) / U(k) \rightarrow U(n) / U(k)
$$

so that on homology

$$
\begin{aligned}
f_{n, k_{*}}=g_{*} \otimes \mathrm{Id}_{*}: H_{*}\left(\mathbb{S}^{2 n-1}\right) \otimes H_{*}(U(n-1) / U(k)) \\
\rightarrow H_{*}\left(\mathbb{S}^{2 n-1}\right) \otimes H_{*}(U(n-1) / U(k)),
\end{aligned}
$$

where $g: \mathbb{S}^{2 n-1} \rightarrow \mathbb{S}^{2 n-1}$ is a map of degree $(n-1)$ !. Thus $\chi^{\text {tor }}\left(f_{n, k}\right)=$ $((n-1) !)^{0}=1$.

The $\mathbb{Z}_{(q)}$-equivalence produced by Proposition 4.3

$$
f: \mathbb{S}^{2 n-1} \times \cdots \times \mathbb{S}^{2 k+1} \rightarrow U(n) / U(k)
$$

is the composite

$$
f=\left(\operatorname{Id}_{\mathbb{S}^{2 n-1}} \times \cdots \times \mathbb{S}^{2 k+5} \times f_{k+2, k}\right) \circ \cdots \circ\left(\operatorname{Id}_{\mathbb{S}^{2 n-1}} \times f_{n-1, k}\right) \circ f_{n, k}
$$

and hence $\chi^{\text {tor }}(f)=1$, and so the result follows from Theorem 4.8(c).

Corollary 4.12. Let $G$ be a subgroup of $U(n)$ which acts on $U(n) / U(k)$ with $k>0$, having rank one isotropy and whose order is prime to $(n-1) !$. Then $G$ acts freely on a finite $C W$-complex having the homotopy type of $\mathbb{S}^{2 n-1} \times \mathbb{S}^{2 n-3} \times$ $\cdots \times \mathbb{S}^{2 k+1} \times \mathbb{S}^{M}$ for some $M>1$. 
Proof. By [2], $G$ acts freely on a finite complex $X$ having the homotopy type of $U(n) / U(k) \times \mathbb{S}^{M}$ for some $M>1$. One then propagates across the map

$$
f \times \text { Id }: \mathbb{S}^{2 n-1} \times \mathbb{S}^{2 n-3} \times \cdots \times \mathbb{S}^{2 k+1} \times \mathbb{S}^{M} \rightarrow U(n) / U(k) \times \mathbb{S}^{M} \simeq X .
$$

The main application is the following intermediate result in the non-simply connected case:

Corollary 4.13. If $G \subset U(n)$ and $|G|$ is prime to $(n-1)$ !, then $G$ acts freely on a finite complex $X \simeq \mathbb{S}^{2 n-1} \times \cdots \times \mathbb{S}^{3} \times \mathbb{S}^{M}$ for some $M>1$.

Proof. It suffices to observe that $G$ will act on $U(n) / U(1)$ with periodic isotropy, whence we can apply the previous corollary.

We do not give the outline of the surgery theoretic proof of Theorem 4.8(d) and (e), but instead refer to the original source [8] which proves the theorem for $p$-groups and to [11] which proves the key fact needed to prove the theorem for general groups $G$.

We are now ready to prove our main theorem by checking the propagation hypotheses.

Proof of Theorem 4.4. Let $X=\mathbb{S}^{2 n-1} \times \mathbb{S}^{2 n-3} \times \cdots \times \mathbb{S}^{2 k+1}$ and $Y=U(n) / U(k)$ and let $f: X \rightarrow Y$ be the map produced by Proposition 4.3. We have seen that conditions (1), (2), and (3) of Theorem 4.4 hold; we only need to verify condition (4).

The definition of the local normal invariant $\nu_{(q)}(f) \in[Y, F / O]_{(q)}$ is quite subtle (see [13, p. 12] for a definition), but we will sidestep the subtleties by noting that $[Y, F / O]_{(q)} \rightarrow[Y, F / O]_{(0)}$ is injective, since $H_{*}(Y ; \mathbb{Z})$ is torsion-free. Since $F / O \rightarrow B O$ is a rational equivalence [22], it suffices to show that the image of the local normal invariant in $[Y, B O]_{(0)}$ is trivial. Its image in $[Y, B O]_{(0)}$ is given by the difference of $\left((\operatorname{deg} f)\left(f_{(0)}^{-1}\right)^{*} \tau_{X}\right)-\tau_{Y}$. But the tangent bundle $\tau_{X}$ is stably trivial, and, according to [29], $\tau_{Y}$ is trivial.

Given a group of rank equal to $r$, a challenging open problem is to construct a free action of $G$ on a finite complex with the homotopy type of a product of $r$ spheres (see [2]). Constructing free actions on actual products of spheres is of course the final goal, and progress on this beyond spherical space forms has been very scant. Indeed, we still cannot verify if every finite group acts freely and homologically trivially on some product of spheres. The following application of Theorem 4.4 provides an infinite number of new examples.

Corollary 4.14. Let $P$ denote a finite non-abelian p-group with cyclic center and an abelian maximal subgroup. If the rank of $P$ is $r$ and $r<p$, then there exists a 
free, smooth and homologically trivial action of $P$ on $M=\mathbb{S}^{2 p-1} \times \cdots \times \mathbb{S}^{2(p-r)+1}$, a product of $r$ spheres.

Proof. Given a group of this type, we know from the results in $\S 2$ that there is a faithful irreducible representation $P \subset U(p)$ of fixity equal to $r-1$. Hence $P$ acts freely on $U(p) / U(p-r)$. Using the propagation theorem, we infer that in fact $P$ must act freely and smoothly on the stated product of spheres.

Example 4.15. Let $P$ denote any $p$-group of fixity equal to one, where $p$ is an odd prime. Then $P$ has a faithful $p$-dimensional representation such that $P$ acts freely on $U(p) / U(p-2)$; hence it will act freely and smoothly on $M=\mathbb{S}^{2 p-1} \times \mathbb{S}^{2 p-3}$. Note that this provides an action on a lower dimensional manifold than that provided by Theorem 3.1.

Example 4.16. Let $P=\mathbb{Z} / p \prec \mathbb{Z} / p$ (the wreath product); this is a group of order $p^{p+1}$, with cyclic center and having a maximal subgroup which is abelian, $(\mathbb{Z} / p)^{p}$. Indeed, there is a natural embedding $P \subset U(p)$, where the elementary abelian subgroup is mapped to the diagonal matrices and the $\mathbb{Z} / p$ action is represented by a permutation matrix. Applying Corollary 4.13, we obtain that $P=\mathbb{Z} / p \nmid \mathbb{Z} / p$ acts freely on a finite complex with the homotopy type of a product of $p$ spheres, namely $X \simeq \mathbb{S}^{2 p-1} \times \cdots \times \mathbb{S}^{3} \times \mathbb{S}^{M}$ for some $M>1$.

\section{References}

[1] A. Adem and J. F. Davis, Topics in transformation groups, in: Handbook of geometric topology, 1-54, North-Holland, 2002.

[2] A. Adem and J. H. Smith, Periodic complexes and group actions, Ann. of Math. 154 (2001), 407-435.

[3] N. Blackburn, Generalizations of certain elementary theorems on p-groups, Proc. London Math. Soc. (3) 11 (1961), 1-22.

[4] H. F. Blichfeldt, Finite Collineation Groups, University of Chicago Press, 1917.

[5] R. Bott, The space of loops on a Lie group, Michigan Math. J. 5 (1958), 35-61.

[6] A. K. Bousfield and D. M. Kan, Localization and completion in homotopy theory, Bull. Amer. Math. Soc. 77 (1971), 1006-1010.

[7] A. K. Bousfield and D. M. Kan, Homotopy limits, completions and localizations, Lecture Notes in Mathematics 304, Springer, 1972.

[8] S. Cappell and S. Weinberger, Homology propagation of group actions, Comm. Pure Appl. Math. 40 (1987), 723-744.

[9] S. B. Conlon, p-groups with an abelian maximal subgroup and cyclic center, J. Austral. Math. Soc. Ser. A 22 (1976), 221-233.

[10] J. Conway et al., Atlas of Finite Groups, Oxford University Press, 1985.

[11] J. F. Davis and P. Löffler, A note on simple duality, Proc. Amer. Math. Soc. 94 (1985), $343-347$.

[12] J. F. Davis and S. Weinberger, Group actions on homology spheres, Invent. Math. 86 (1986), 209-231.

[13] J. F. Davis and S. Weinberger, Obstructions to propagation of group actions, Bol. Soc. Mat. Mexicana (3) 2 (1996), 1-14. 
[14] R. Dotzel and G. C. Hamrick, p-group actions on homology spheres, Invent. Math. 62 (1981), 437-442.

[15] W. Feit, The current situation in the theory of finite simple groups, in: Actes du Congrès International des Mathématiciens (Nice, 1970), Tome I, 55-93, Gauthier-Villars, 1971.

[16] A. Heller, A note on spaces with operators, Illinois J. Math. 3 (1959), 98-100.

[17] P. Hilton, G. Mislin and J. Roitberg, Localization of nilpotent groups and spaces, NorthHolland Mathematics Studies, No. 15, North-Holland Publishing Co., 1975.

[18] B. Huppert, Endliche Gruppen I, Grundlehren vol. 134, Springer-Verlag, Berlin, 1967.

[19] D. Husemoller, Fibre bundles, Graduate Texts in Mathematics 20, Springer-Verlag, 1966.

[20] G. Lewis, Free actions on $\mathbb{S}^{n} \times \mathbb{S}^{n}$, Trans. Amer. Math. Soc. 132 (1968), 531-540.

[21] W. Lück, Transformation groups and algebraic K-theory, Lecture Notes in Mathematics 1408, Springer-Verlag, 1989.

[22] I. Madsen and R. J. Milgram, The classifying spaces for surgery and cobordism of manifolds, Annals of Mathematics Studies 92, Princeton University Press, 1979.

[23] I. Madsen, C. B. Thomas and C. T. C. Wall, The topological spherical space form problem. II. Existence of free actions, Topology 15 (1976), 375-382.

[24] M. F. Newman, E. A. O'Brien and A. Shalev, The fixity of groups of prime-power order, Bull. London Math. Soc. 27 (1995), 225-231.

[25] D. Quillen, The spectrum of an equivariant cohomology ring I, Ann. of Math. 94 (1971), 549-572.

[26] U. Ray, Free linear actions of finite groups on a product of spheres, J. Algebra 147 (1992), $456-490$.

[27] D. S. Rim, Modules over finite groups, Ann. of Math. 69 (1959), 700-712.

[28] A. Shalev, On the fixity of linear groups, Proc. London Math. Soc. (3) 68 (1994), 265-293.

[29] W. A. Sutherland, A note on the parallelizability of sphere-bundles over spheres, J. London Math. Soc. 39 (1964), 55-62.

[30] M. Suzuki, Group Theory II, Grundlehren vol. 248, Springer-Verlag, New York, 1986.

[31] Ö. Ünlü, UW-Madison Doctoral Dissertation, in preparation.

[32] C. T. C. Wall, Finiteness conditions for CW-complexes, Ann. of Math. 81 (1965), 56-69.

[33] S. Weinberger, Homologically trivial group actions. I. Simply connected manifolds, Amer. J. Math. 108 (1986), 1005-1021.

[34] G. W. Whitehead, Elements of homotopy theory, Graduate Texts in Mathematics 61, Springer-Verlag, 1978.

[35] N. Yagita, On the dimension of spheres whose product admits a free action by a non-abelian group, Quart. J. Math. Oxford 36 (1985), 117-127.

A. Adem

Department of Mathematics

University of Wisconsin

Madison WI 53706

USA

e-mail: adem@math.wisc.edu

Ö. Ünlü

Department of Mathematics

University of Wisconsin

Madison WI 53706

USA

e-mail: unlu@math.wisc.edu

\section{J. F. Davis}

Department of Mathematics

Indiana University

Bloomington IN 47405

USA

e-mail: jfdavis@indiana.edu

(Received: April 28, 2003) 